\title{
The value of patient feedback in the audit of TMJ arthroscopy
}

\author{
D. R. P. Godden, ${ }^{1}$ and J. M. Robertson, ${ }^{2}$
}

\begin{abstract}
Aim This study assessed the patients' and clinicians' perception of the outcome of temporomandibular joint arthroscopy.

Method All patients who underwent TMJ arthroscopy for both diagnostic and therapeutic purposes over a 6-year period were sent a questionnaire that asked about various symptoms attributable to the TMJ. Additionally a review of the clinical notes was performed.

Results 83 patients underwent arthroscopy to 127

temporomandibular joints. The mean follow up was 3.6 years.

$55 \%$ of patients assessed their jaw function as being effective, jaw movement, pain control, and overall satisfaction were satisfactory in $37 \%, 57 \%$, and $48 \%$ of cases respectively. The clinicians' assessment revealed that $45 \%$ of patients had no joint tenderness, $74 \%$ of patients were able to open to $>35 \mathrm{~mm}$ and $74 \%$ of patients were free of any joint noise. $66 \%$ of patients were prepared to undergo a second procedure if indicated.

Conclusion Overall, $50 \%$ of patients seemed to view arthroscopy favourably although many patients still felt that jaw opening was restricted. The outcome was not related to the position and reducibility of the disc at surgery and other variables may be responsible. The disparity between the clinical evaluation and the patients' perception of effectiveness emphasises the importance of patient feedback.
\end{abstract}

Tternal derangement of the temporomandibular joint (TMJ) is a common problem in dental practice. The role of surgery in its management is controversial and the indications are relative rather than absolute. ${ }^{1}$ Similarly the definition of success in surgery of the TMJ is difficult to quantify, although guidelines are available. ${ }^{2}$ The use of an arthroscopic approach to the TMJ has become widely used in the management of TMJ disorders. It provides minimally invasive access to the joint and while initially it was used as a diagnostic tool, more recently with the refinement of instrumentation it has been used therapeutically and success rates following treatment range from $50-100 \% .^{3-16}$ Patients with painful limited opening (closed lock) generally fare better than those with normal opening. $3,5,7,10,16$ The reason for this is not clear, simple lavage and lysis of the superior joint space without formal disc repositioning is successful, and consequently the importance of disc position has been questioned..$^{8,9,17,18}$ Kircos has shown disc displacement in 30\% of asymptomatic volunteers following MR scanning. ${ }^{18}$ The identification of the various chemical mediators of inflammation in the syn-

\footnotetext{
${ }^{1}$ Specialist Registrar, Department of Oral and Maxillofacial Surgery, Peterborough District Hospital, Thorpe Road, Peterborough PE3 6DA;

${ }^{2}$ Consultant, Department of Oral \& Maxillofacial Surgery, Peterborough District Hospital, Thorpe Road, Peterborough PE3 6DA

Correspondence to: Mr D R P Godden, 3 Oak Coppice, Bolton BL1 5JD

REFEREED PAPER

Received 19.04.99; accepted 13.10.99

(C) British Dental Journal 2000; 188: 37-39
}

ovial fluid may account for many of the symptoms and may explain why arthrocentesis or arthroscopic wash out work. ${ }^{19}$ Despite continuing research into the efficacy of arthroscopy and despite the fact that there is a wealth of information from the clinician's view, there is little detail focused on the patients' perception of its success. The aim of this study was to evaluate the success of arthroscopy by means of both a patient questionnaire and a retrospective clinical notes review. In addition, a comparison was made between the patients who were found to have an irreducibly displaced meniscus and those who had evidence of degenerative change only, to investigate which group did best.

\section{Method}

This study was set in the Oral Surgery Department at Peterborough District Hospital. All patients who underwent arthroscopy to the TMJ between 1991 and 1996 were sent a questionnaire during 1997. The format of this is described by Fridrich et al. ${ }^{3}$ It subjectively evaluated the perceptions of pain, joint noise, jaw mobility and function. It asked the patients whether they considered their surgery a success and whether they were prepared to undergo surgery again if required. The questions were in the form of a visual analogue scale (VAS). To facilitate an assessment of the success of surgery, patients have been placed into bands according to the score on the visual analogue scale. The definition of success or failure is very much an arbitrary judgement. We have chosen success if the score was within $75 \%$ of normal and failure if the patient scored within $25 \%$ of grossly abnormal. Patients who scored the VAS between these values are described as borderline.

The clinical notes review recorded the presence of joint tenderness, the degree of mouth opening and the presence of joint noise. In addition, the arthroscopic findings were recorded and these were compared with the patients' perception of outcome.

Prior to arthroscopy all patients had received a period of conservative management including, where indicated, the use of analgesics, tricyclic antidepressants and bite raising appliances. Arthroscopy was carried out when there was a significant degree of pain, hypomobility, or joint noise. Dual port arthroscopy followed by lysis and lavage and instrument sweep of the superior joint space was performed. Intra-articular triamcinalone was infused at the end of the procedure where significant synovial inflammation was found. Patients were discharged with instructions to maintain a soft diet for 1 week and to encourage active mouth opening with exercises after 24 hours.

Statistical analysis was performed using SPSS statistics package on PC, statistical significance was determined at the 5\% level.

\section{Results}

During the study period a total of 110 patients underwent arthroscopy. Ten patients could not be contacted by post because of change of address. Of the remaining 100 patients, 83 patients responded to the questionnaire. There were 68 female and 15 male 
Fig. 1 Patient satisfaction 'rate your level of satisfaction with the operation' $n=80$, three patients failed to score their satisfaction with the surgery, mean $=59.7,0=$ total failure $100=$ complete success

\section{Number of patients}

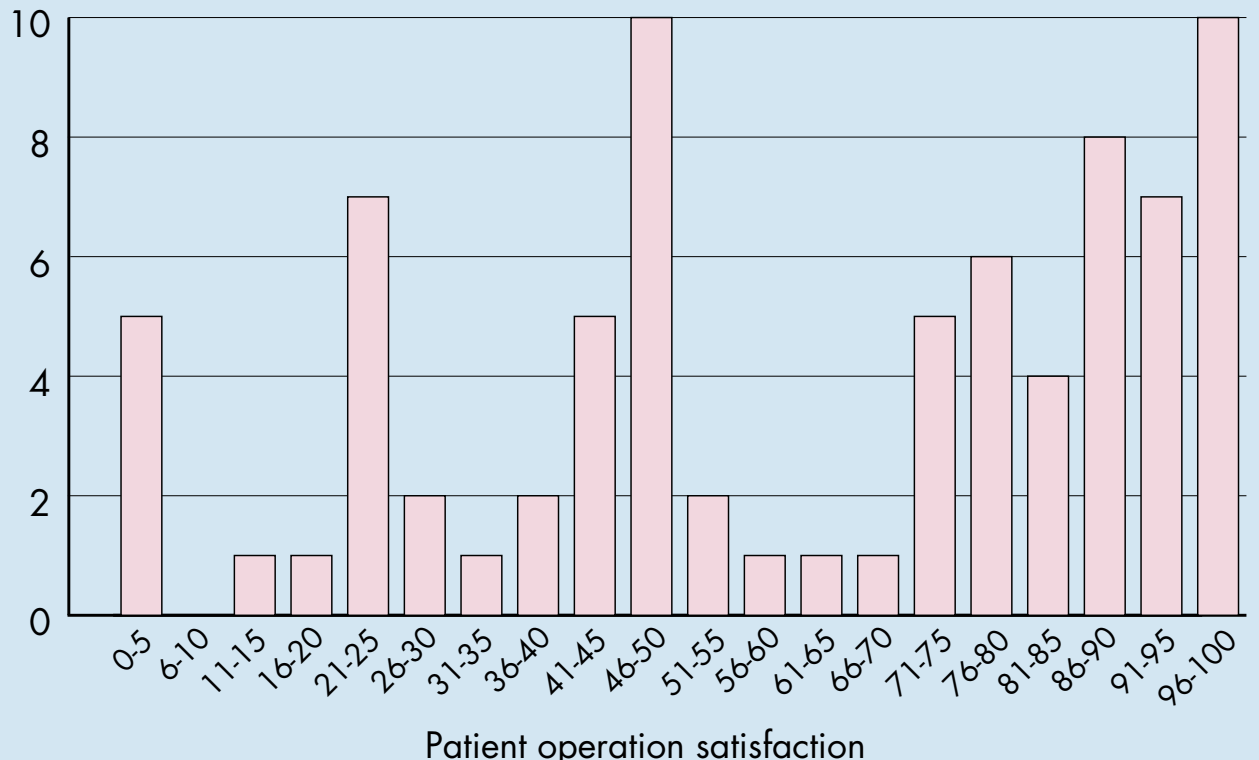

patients. The mean age at surgery was 34.7 years (range 17-75 years). The mean follow-up period from surgery to questionnaire was 3.6 years. Thirty-nine patients underwent unilateral arthroscopy; 44 patients had a bilateral procedure, a total of 127 joints were arthroscoped. Five patients $(6 \%)$ required subsequent re-arthroscopy. The median conservative management period prior to surgery was 5.5 months.

The responses received from the patients were clustered in the middle and toward the satisfactory end of the scale, creating a bimodal distribution for all the parameters recorded. This is shown by the overall satisfaction response in Figure 1. Fifty-five per cent of patients rated jaw function as being effective; jaw movement, pain control, and overall satisfaction were in the satisfactory range for $37 \%, 57 \%$ and $48 \%$ of cases respectively (Table 1 ).

When the patients were asked if they were prepared to undergo further arthroscopy again if warranted, 55 patients $(66 \%)$ said yes, 8 patients (10\%) said no and the remainder $(24 \%)$ did not know.

At surgery 38 patients were found to have irreducible disc displacement and 24 patients had degenerative changes. Degenerative change includes adhesions, fibrillation, and chondromalacia. The remaining 21 patients $(25 \%)$ had normal or mixed findings during a bilateral procedure. There was no statistical difference in the VAS responses for any of the parameters measured between the two groups (Mann-Whitney $U$ Test, $P>0.05$ ) (Table 2).

The clinical notes review revealed that following arthroscopy there was an improvement in all the parameters recorded. The absence of joint noise improved from $22 \%$ to $74 \%, 45 \%$ were free of joint tenderness and $73 \%$ of patients could open to $>35 \mathrm{~mm}$ following arthroscopy (Table 3).

\section{Discussion}

The success of arthroscopy is difficult to quantify accurately. The criteria have been examined by Goss. ${ }^{2}$ The absence of pain is an ideal criterion, but a satisfactory outcome may be achieved if the pain is significantly reduced in both intensity and frequency. The range of opening should be $35 \mathrm{~mm}$ or more. While this is still restricted, it correlates well with a subjective feeling of well-being. The absence of joint sounds is an ideal criterion, however, it is frequently surgically unobtainable. Joint sounds are so common in the population that they can be considered a normal finding. Finally the patient should
Table 1 VAS scores for all patients

\begin{tabular}{lcrrr}
\hline & Mean VAS scores & Success & \multicolumn{1}{l}{ Failure } & Borderline \\
\hline Pain & $28.7^{\dagger}$ & $47(57 \%)$ & $4(5 \%)$ & $32(39 \%)$ \\
Noise & $34.3^{\ddagger}$ & $43(52 \%)$ & $8(10 \%)$ & $32(39 \%)$ \\
Movement & $36.5^{\alpha}$ & $31(37 \%)$ & $5(6 \%)$ & $47(57 \%)$ \\
Function & $27.4^{\beta}$ & $46(55 \%)$ & $2(2 \%)$ & $35(42 \%)$ \\
Satisfaction* & $59.7^{*}$ & $38(48 \%)$ & $17(21 \%)$ & $25(31 \%)$ \\
\hline
\end{tabular}

$n=83,{ }^{*} n=80,{ }^{\dagger} 0=$ no pain, $100=$ severe pain; ${ }^{\dagger} 0=$ no noise, $100=$ extremely loud noise; ${ }^{\alpha} 0=$ no restriction, $100=$ extreme restriction; $\beta 0=$ no restriction to chewing, $100=$ liquid diet, unable to chew solids; $\gamma 0=$ total failure, $100=$ complete success

Table 2 Mean VAS scores according to disc position

\begin{tabular}{lccc}
\hline & $\begin{array}{c}\text { Degenerative change } \\
\text { mean value }\end{array}$ & $\begin{array}{c}\text { Irreducible disc } \\
\text { mean value }\end{array}$ & $P$-value* \\
\hline Pain & 31 & 26 & 0.36 \\
Noise & 39 & 31 & 0.23 \\
Movement & 36 & 32 & 0.50 \\
Function & 30 & 21 & 0.14 \\
Satisfaction ${ }^{\dagger}$ & 58 & 63 & 0.52
\end{tabular}

${ }^{*}$ Mann Whitney $U$ test, $n=83,{ }^{\dagger} n=80$

Table 3 Clinical evaluation pre- and post-arthroscopy

\begin{tabular}{lrr}
\hline & Pre-arthroscopy & Post-arthroscopy \\
\hline Joint tenderness & $7(8 \%)$ & $37(45 \%)$ \\
Nil & $63(76 \%)$ & $38(46 \%)$ \\
Mild & $13(16 \%)$ & $8(10 \%)$ \\
Severe & $42(51 \%)$ & $61(73 \%)$ \\
\hline Jaw opening $>35 \mathrm{~mm}$ & & \\
\hline & & \\
Jaw noise & $18(22 \%)$ & $15(18 \%)$ \\
Nil & $58(70 \%)$ & $7(8 \%)$ \\
Soft & $7(8 \%)$ & \\
Loud & &
\end{tabular}

$n=83$ 
be able to achieve full jaw function without any restrictions. When the measures of pain and jaw function are used, success ranges from $50 \%-100 \% .^{3,5-16}$ Caution has to be exercised in any interpretation, as these studies often contain only small patient numbers, have limited follow up, and use differing criteria for success.

With experience in the use of arthroscopy it has become apparent that patients with painful limited opening (closed lock) do better when compared with patients who are able to achieve wider opening. $3,5,7,9,10,15,16$ The reason for this is not clear. The therapeutic success of arthroscopy may be caused by the removal of disc adhesions, manipulation of the mandible during the procedure, joint wash out or the use of intracapsular steroid injection at the end of surgery. ${ }^{15}$ Equally successful outcomes can be achieved by simple arthrocentesis of the joint. ${ }^{3}$

In the analysis of these results it is apparent that for all parameters there was a bimodal distribution, suggesting that there are two groups of patients within the sample. There is a fairly consistent group of patients who did well in all the parameters recorded, and a second group who tended to cluster in the middle. The reason for this is not clear. It is recognised that respondents tend to score VAS scales in the middle of the scale and avoid extremes. This would introduce an element of bias. The distribution pattern did not seem to be influenced by the reducibility of the disc. Other variables which may influence outcome but which have not been studied here include the degree of trauma on entry to the joint, age of the patient, and psychiatric status. These variables may introduce flaws into the methodology. The response rate for the questionnaire was high at $83 \%$, and the sample size of 100 patients was adequate.

Analysis of the results reveals that while the clinical assessment showed that $73 \%$ of patients were able to open to greater than $35 \mathrm{~mm}$, the questionnaire found that only $37 \%$ rated jaw movement as in the satisfactory range, suggesting that $35 \mathrm{~mm}$ is not enough. In addition the clinical review indicated that $90 \%$ of patients had no or only mild joint tenderness yet only $57 \%$ recorded that they had satisfactory pain control on self-assessment. This highlights the fact that there is a disparity between the impression of the clinician and the perception of the patient. The routine use of a VAS in clinical practice would facilitate more accurate feedback, and their use in clinical trials reduces the degree of subjectivity in assessment. Of the parameters investigated nearly two-thirds of patients felt dissatisfied with the degree of mandibular movement while pain, joint noise and overall function were considered satisfactory in around one half of cases.

The direct comparison of these results with other studies should be exercised with caution since like groups were not used. The patients in this study received arthroscopy for diagnostic purposes as well as for the therapeutic management of closed lock, they were a much more mixed group than many of the other trials which tend to restrict their investigation to the management of closed lock patients alone. Despite the above, the questionnaire indicates that around one half of patients perceive arthroscopy favourably and two-thirds were prepared to undergo the procedure again. Disc position did not influence outcome and this is in contradistinction to other studies. One reason for this, as suggested by Nitzan et al. ${ }^{9}$ may be that disc position is not as crucial as previously thought. Of the parameters recorded, jaw movement gave the most disappointing result.

The long-term effects of arthroscopy on the TMJ are not known. It is speculated that arthroscopy of the TMJ may be similar to arthroscopy of other joints such as the knee where there is an initial benefit but which is not long lasting. ${ }^{20}$ Because there is experi- mental evidence that degenerative changes can be induced by diagnostic arthroscopy, ${ }^{21}$ and because there is evidence that the natural history of this disorder is that of spontaneous improvement with time, ${ }^{22}$ it would be prudent to restrict arthroscopy to those patients who benefit most. This study has attempted to evaluate the patients' attitude to the effectiveness of arthroscopy. It has shown that there is a disparity between the patients' perceived benefit and the clinician's evaluation. On the face of it, disc position did not influence outcome. To discover who benefits most, further investigation with a control group consisting of patients who are managed conservatively is warranted.

1 Dolwick M F, Dimitroulis G. Is there a role for temporomandibular joint surgery? Br J Oral Maxillofac Surg 1994; 32: 307-313.

2 Goss A N. Towards an international consensus on temporomandibular joint surgery. Int J Oral Maxillofac Surg 1993; 22: 78-81.

3 Fridrich K L, Wise J M, Zeitler D L. Prospective comparison of arthroscopy and arthrocentesis for temporomandibular joint disorders. J Oral Maxillofoc Surg 1996; 54: 816-820.

4 Ohnishi M. Arthroscopy of the temporomandibolar joint. J Jpn Stomat 1975; 42: 207-212.

5 Davis C L, Kaminishi R M, Marshall M W. Arthroscopic surgery for treatment of closed lock. J Oral Maxillofac Surg 1991; 49: 704-707.

6 Gabler M J, Greene C S, Palacios E, Perry H T. Effect of arthroscopic temporomandibular joint surgery on articular disc position. $J$ Craniomandib Disord Facial Oral Pain 1989; 3: 191-202.

7 Indresano A T. Arthroscopic surgery of the temporomandibular joint: report of 64 patients with long-term follow-up. J Oral Maxillofac Surg 1989; 47: 439-441.

8 Moses J J, Sartorius D, Glass R, Tanaka T, Poker I. The effects of arthroscopic surgical lysis and lavage of the superior joint space on the TMJ disc position and mobility. J Oral Maxillofac Surg 1989; 47: 674-678.

9 Nitzan D W, Dolwick M F, Heft M W. Arthroscopic lavage and lysis of the temporomandibular joint: a change in perspective. J Oral Maxillofac Surg 1990; 48: 798-801.

10 Sanders B. Arthroscopic surgery of the temporomandibular joint: treatment of internal derangement with persistent closed lock. Oral Surg Oral Med Oral Pathol 1986; 62: 361-372.

11 Sanders B, Buoncristiani R D. Diagnostic and surgical arthroscopy of the temporomandibular joint: clinical experiences with 137 procedures over a 2-year period. J Craniomand Disorders 1986; 8: 203-213.

12 White R D. Retrospective analysis of 100 consecutive surgical arthroscopies of the TMJ. J Oral Maxillofac Surg 1989; 47: 1014-1028

13 Tarro W. Arthroscopic diagnosis and surgery of the TMJ. J Oral Surg 1988; 46: 282-289.

14 Israel H A, Roser S M. Patient response to temporomandibular joint arthroscopy: preliminary findings in 24 patients. J Oral Maxillofac Surg 1989; 47: 570-573.

15 Clark C T, Mood D G, Sanders B. Arthroscopic treatment of temporomandibular joint locking resulting from disc derangement: Twoyear results. J Oral Maxillofac Surg 1991; 49: 157-164.

16 Holmlund A, Gynsher C, Axelsson S. Efficacy of arthroscopic lysis and lavage in patients with chronic locking of the temporomandibular joint. Int J Oral Maxillofac Surg 1994; 23: 262-265.

17 Dolwick M E, Dimitroulis C. A re-evaluation of the importance of disc position in temporomandibular disorders. Aust Dent J 1996; 41: 184-187.

18 Kircos L T, Ortendahl D A, Mark A S, et al. Magnetic resonance imaging of the TMJ disc in asymptomatic volunteers. J Oral Maxillofac Surg 1987; 45: 397-401.

19 Quinn J H. Identification of Prostaglandin E2 ond Leokotriene B4 in the synoviol fluid of painful, dysfuntional temporomandibular joints. J Oral Maxillofac Surg 1990; 48: 968-971.

20 Livesly P S, Doherty M, Needoff M, Malton A. Arthroscopic lavage of osteoarthritic knees. J Bone Joint Surgery (Br) 1991; 73B: 922-926.

21 Bjornland T, Rorvik M, Haanoes B R, Teige J. Degenerative changes in the temporomandibular joint after diagnostic arthroscopy. Int J Oral Maxillofac Surg 1994; 23: 41-45.

22 Sato S, Sakamoto M, Kawamura H, Motegi K. Long-term changes in clinical signs and symptoms and disc position and morphology in patients with nonreducing disc displacement in the temporomandibular joint. $J$ Oral Maxillofac Surg 1999; 57: 23-29. 